\title{
New Strategic Plan Takes the ALS into the Future
}

\author{
J. Kirz ${ }^{* \dagger}$, D.S. Chemla*, B. Feinberg ${ }^{*}$, Z. Hussain, G.F. Krebs ${ }^{*}$, H.A. Padmore ${ }^{*}$, \\ D.S. Robin",A.L. Robinson ${ }^{*}$, N.V. Smith", and T. Warwick ${ }^{*}$ \\ *Advanced Light Source, Lawrence Berkeley National Laboratory, Berkeley, CA 94720 USA \\ ${ }^{\dagger}$ Department of Physics and Astronomy, Stony Brook University, Stony Brook, NY 11794 USA
}

\begin{abstract}
A new strategic plan is in place to upgrade the ALS so it can continue to address fundamental questions, such as size-dependent and dimensional-confinement phenomena at the nanoscale; correlation and complexity in physical, biological, and environmental systems; and temporal evolution, assembly, dynamics and ultrafast phenomena. Moreover, the growing number of ALS users (now exceeding 2,000 per year) requires increased attention. Accordingly, our plan concentrates on projects that will continue to make it possible for ALS users to address grand scientific and technological challenges with incisive world-class tools and quality user support. Our highest priority is to begin top-off operation, in which electrons are injected into the storage ring at intervals of approximately 1 minute. The combination of top-off and concurrent development of small-gap in-vacuum undulators and superconducting undulators will allow an increase in brightness from eight to more than 100 times, depending on the specific undulators and photon energy range. As part of our core mission in the VUV and soft x-ray regions, we plan to exploit these accelerator developments to extend our capabilities for high spatial and temporal resolution and utilize the remarkable coherence properties of the ALS in a new generation of beamlines. Ranked by priority, several proposed beamlines will follow completion of five new beamlines already under construction or funded. The intellectual excitement of the ALS has been a powerful tool in the recruitment and retention of outstanding staff, but additional sustained efforts are required to increase diversity both in gender and in underrepresented groups. To this end, we intend to expand the ALS Doctoral Fellowship Program by giving special emphasis to underrepresented groups. We also envision a distinguished postdoctoral fellowship program with the same emphasis, to increase and diversify our pool of candidates for beamline scientist positions.
\end{abstract}

Keywords: Synchrotron radiation, insertion devices, $x$-ray optics, $x$-ray instrumentation,

PACS: 07.85.Qe, 07.85.Fv, 41.50.+h, 29.20.Lq

\section{INTRODUCTION}

The ALS stands at a pivotal point in its history. The available straight sections are built out or spoken for, and only a few of the superbend lines remain uncommitted. The emphasis of the new strategic plan is to keep the ALS at the cutting edge for the next 2-3 decades by honing our existing stock and by creating sharper tools that exploit the very significant advances in accelerator and insertion-device technology that have occurred since the ALS began operation in 1993. Now underway, the strategic plan upgrades the ALS so it can continue to address fundamental questions, such as size-dependent and dimensional-confinement phenomena at the nanoscale; correlation and complexity in physical, biological, and environmental systems; and temporal evolution, assembly, dynamics, and ultrafast phenomena. The planning process is ongoing, with further workshops and reviews planned to validate the scientific need, organize the teams, and create detailed designs for each major component of the plan [1].

\section{TOP-OFF IS THE HIGHEST PRIORITY}

Our highest priority is to establish top-off operation of the storage ring, with $500 \mathrm{~mA}$ current. We expect to benefit from a $100 \%$ increased time-averaged current, and much-improved stability of the beam due to a constant heat load on the accelerator vacuum chamber and, especially, on beamline optics. At our lower-energy ring, lifetimes are limited by the Touschek effect. To keep the lifetime reasonable in our normal operations, the ALS is running at a considerably lower brightness than its full capability. 
With top-off, this compromise will no longer be necessary, and the vertical emittance of the beam will decrease almost eightfold, thus providing an increase in brightness. The improvement in the absolute stability of the beam with top-off becomes even more important for the resulting smaller source size. Top-off requires upgrading the booster from 1.5 $\mathrm{GeV}$ to $1.9 \mathrm{GeV}$ operation, significant changes to the radiation-protection system, and a variety of other hardware and control improvements. The detailed design was completed and validated by an external review committee in November 2004. We will upgrade to a fullenergy injector in a fall-2006 shutdown. After the shutdown, we will gradually migrate to topoff injection, with higher currents and smaller emittance.

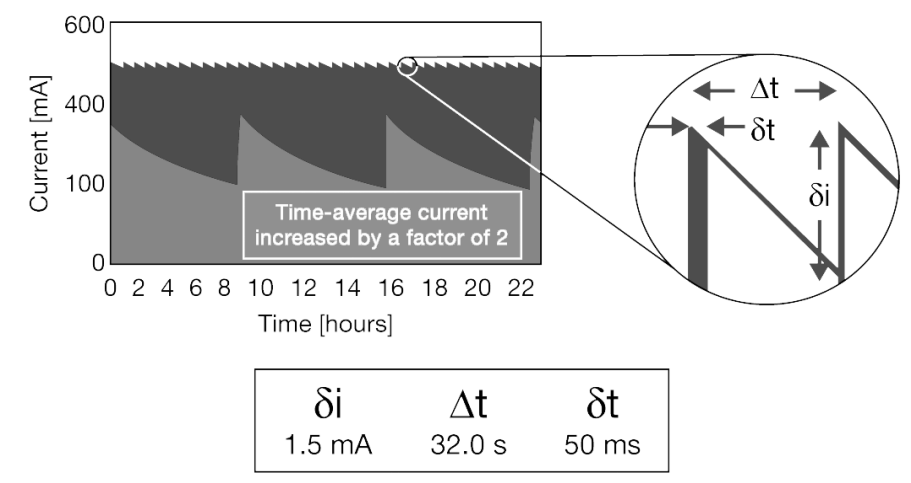

FIGURE 1. Top-off will enhance brightness by doubling the timeaveraged beam current and permitting operation with a lower vertical emittance. It will also result in a constant heat load on the accelerator vacuum chamber and the beamline optics.

\section{ADVANCED INSERTION DEVICES ENHANCE BRIGHTNESS}

The ALS "workhorse" insertion device has been a 4.45-m-long, 50-mm-period linearly polarizing undulator that fills one storage-ring straight section. The ALS has five of these insertion devices, which were designed and installed about a decade ago. In the meantime, undulator technology has undergone significant advances, and we now also have three two-meter elliptically polarizing undulators (EPU) and more proposed. In addition, we are now operating with the first of a number of planned small-gap in-vacuum undulators, further enhancing the brightness, especially in the important energy range above $500 \mathrm{eV}$. Concurrent development of $\mathrm{Nb}_{3} \mathrm{Sn}$-based superconducting undulators will extend the reach of ALS undulators, especially in the multi-keV range. The new undulators allow us to replace the full-length linearly polarized undulators with straight sections containing two slightly offset, shorter, more advanced devices. Not only will these devices perform far better than the current devices, but they will allow simultaneous operation of two fully optimized, independent, application-specific beamlines in each straight section.

\section{NEW APPLICATION-SPECIFIC BEAMLINES AND UPGRADES}

Among a new generation of beamlines that will address the outstanding problems of the coming decades, five beamlines, three with advanced undulators, are already under construction, with some nearing completion.

- EPU-based Beamline 11.0.1 will soon house PEEM-3, a third-generation photoemission electron microscope with a spatial resolution of a few nanometers

- Based on a recently installed 3-cm-period in-vacuum undulator, the ultrafast x-ray facility (Beamlines 6.0.1, 6.0.2) is nearly ready for the study of atomic motion at the molecular-vibration time scale.

- MERLIN (4.0.1) is a EPU beamline designed to provide ultrahigh-energy resolution for ARPES and IXS. It will operate in the VUV energy range below $150 \mathrm{eV}$ to study low-energy excitations in strongly correlated systems.

- Based on a new full-field-imaging x-ray microscope (XM-2), bend-magnet Beamline 2.1 for high-resolution soft X-ray microscopy and tomography will part of the new National Center for X-Ray Tomography.

- Our highly successful microdiffraction program is migrating from its bend-magnet beamline (7.3.3) to a new home on a superbend (12.3.2).

With top-off, we will be able to replace our 11-year-old long undulators with pairs of slightly offset 2-m-long modern insertion devices with superior performance. The first wave of these proposed beamlines includes:

- Straight section 7 is our highest priority. We propose to establish in half of straight section 7 a beamline (COSMIC) to provide coherent light in the $0.25-2.5 \mathrm{keV}$ range with full polarization control and three branches, one for soft x-ray coherent scattering, the second for diffraction microscopy (a new form of lensless imaging designed to provide three-dimensional structures to $10-\mathrm{nm}$ resolution or higher), and the third for substantial upgrades to our existing flagship resonant inelastic x-ray scattering (RIXS) facility.

- In the other half of straight section 7, we propose MAESTRO. In one branch, a "nanoARPES" facility for the study of surfaces and structures created in the Molecular Foundry (Berkeley Lab's new nanoscience center), the 


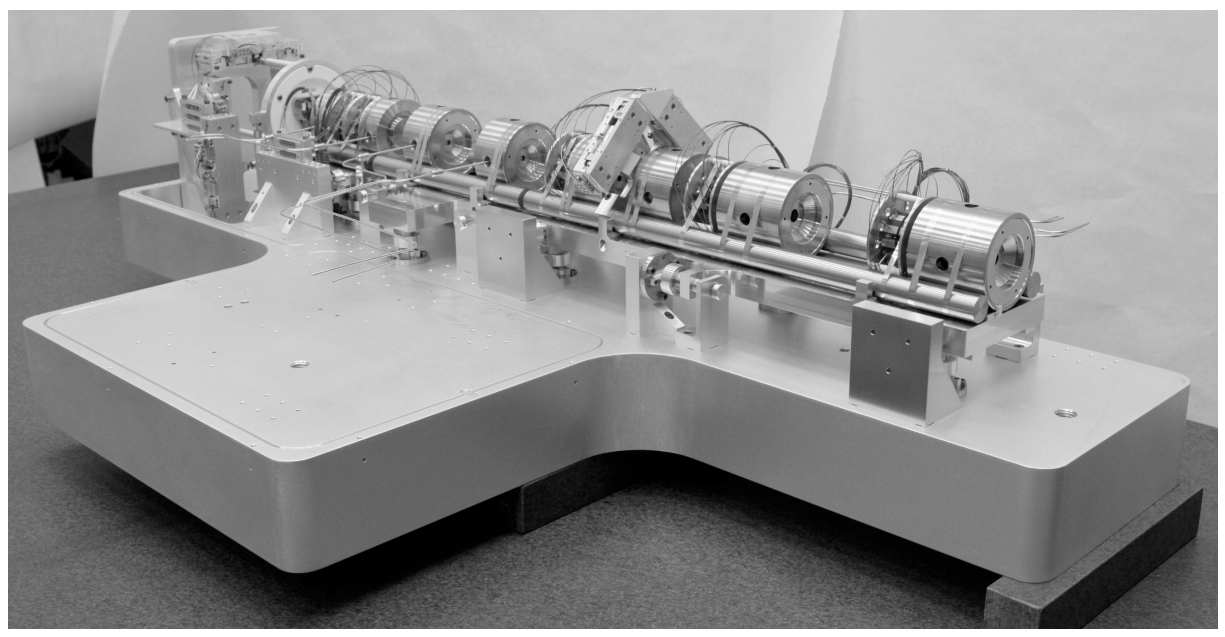

FIGURE 2. PEEM 3 sample manipulator and electron optic train is one of three main components that are now assembled and ready for installation in anticipation of startup in spring 2006.

many interesting materials that are inhomogeneous (i.e., in nanoscale phase segregations), or ultrasmall samples that cannot be prepared in large formats. The other branch of MAESTRO will be devoted to an upgrade of the existing facilities to perform microARPES.

- Second priority is a pair of new EPU beamlines in straight section 8 for soft $\mathrm{x}$-ray scattering and photonin/photon-out techniques. This facility will make it possible to follow, in real-time and with elemental discrimination, the synthesis and self-assembly of novel nanostructures. This beamline will facilitate the development of joint programs with the recently completed Molecular Foundry and Beamlines 11.3.1 (smallmolecule crystallography) and 10.3.2 (micro-XAS).

We need to keep our over subscribed flagship beamlines at the cutting edge. The highest priority is to divide the Sector 10 straight section into two offset sections and give each branch-the photoemission branch and the atomic/molecular physics branch - a separate EPU for full polarization control and a separate beamline so they can run simultaneously. Each of the new, separate beamlines will be application-specific, optimized for the scientific program of the two user communities, without the need for the compromises of the shared beamline in current use.

For studies of both basic and technologically important problems involving domain switching, vortex dynamics, and exchange interactions, we plan to place an EPU in the empty half of straight section 6 to be time-shared between a new, dedicated program in ultrafast magnetism and the soft x-ray branch of the slicing source. This new facility will focus a confluence of experimental and computational interest into problems that overlap synergistically with the mission of the Molecular Foundry and the community that is coalescing around ultrafast phenomena in general.

While MERLIN is designed for the ultimate energy resolution below $150 \mathrm{eV}$, there is a great need to extend the capability for ultrahigh-resolution IXS investigations to energies at least as high as $1 \mathrm{keV}$, so as to be able to scan the momentum (q) vector out to the Brillouin zone boundary. We plan to design a q-resolved inelastic scattering beamline (QERLIN), which should fit into Sector 2 with a modest rearrangement of accelerator components. The incorporation of very high resolution photoemission in the $1-\mathrm{keV}$ range would also be considered.

\section{DETECTORS, SUPPORT FACILITIES, AND BEAMLINE SCIENTISTS}

Available detectors are not well-matched to the capabilities of the ALS and other high-brightness synchrotron radiation sources. There is need for a broad-based program, based in part on high-energy-physics detector developments, to create high-rate pixel detectors. In addition, high-speed streak cameras are needed to fully utilize the femtosecond sources coming online. The ALS, in cooperation with the Berkeley Lab Engineering Division, plans to expand detector development and bring it to successful conclusion via technology transfer to vendors.

Both safety and productivity are jeopardized when crowding becomes extreme, when there is inadequate lab and office space available to support the program. Berkeley Lab has designed a User Support Building that will replace an older building whose occupants are now being relocated in anticipation of demolishment. This is a very high priority for the ALS. The ALS has also undertaken an on-site housing project. It is well under way with the site located and a projected fall 2008 completion for construction of a 60-room, three-story facility next to the ALS. 


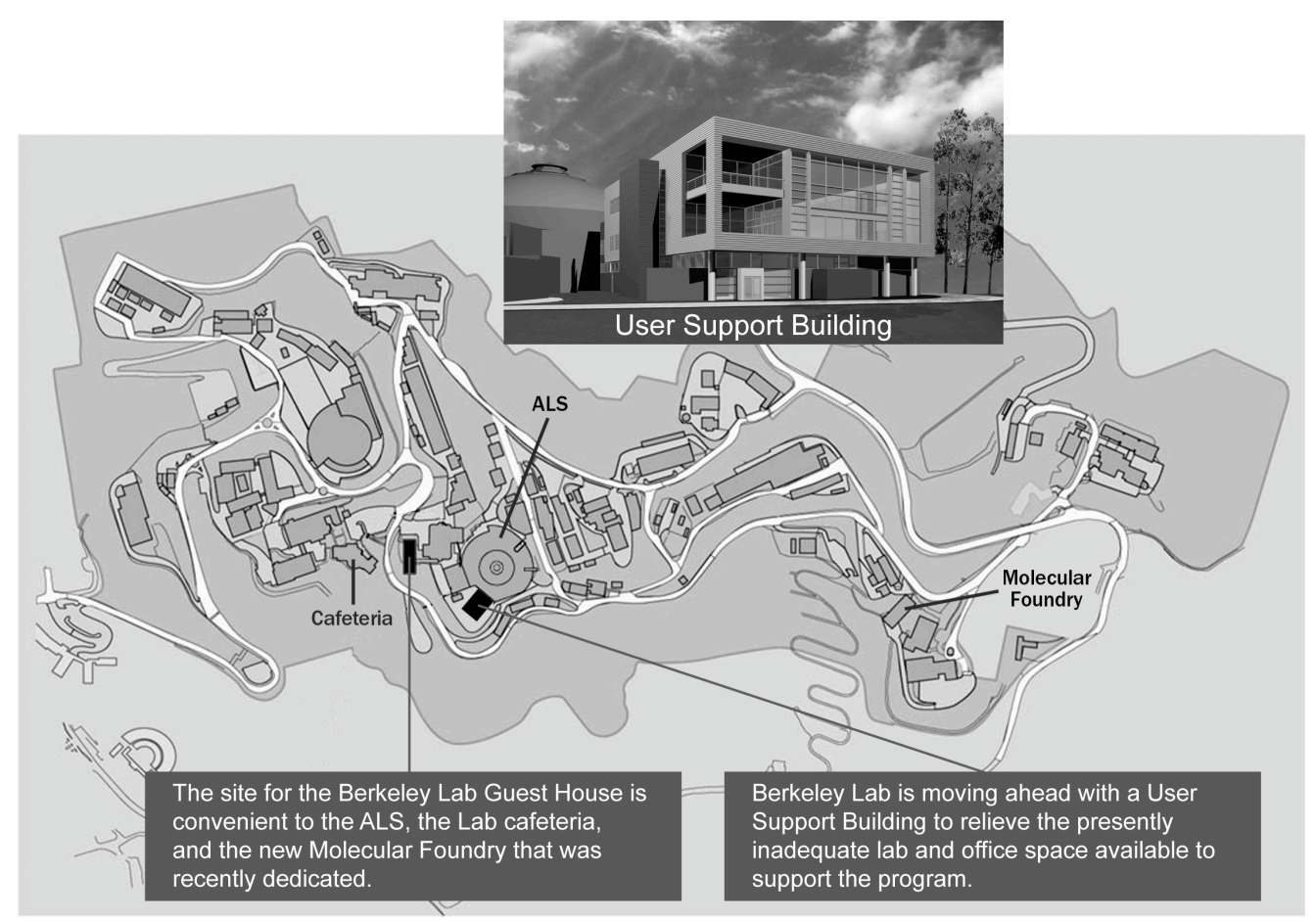

FIGURE 3. New support facilities in the pipeline.

The intellectual excitement of the ALS as a forefront science facility has been a powerful tool in the recruitment and retention of outstanding staff. However, the staffing level of most of the beamlines is barely half of what is considered optimal. We are simultaneously attempting to increase beamline staff in order to optimize utilization of the facility and increase diversity both in gender and in underrepresented groups. The ALS will expand its Doctoral Fellowship Program and also envisions a distinguished postdoctoral fellowship program to increase and diversify the candidate pool for beamline scientist positions.

\section{THE MEDIUM AND FAR FUTURE: COHERENT THz AND ACCELERATOR R\&D}

Two years ago, a national workshop on $\mathrm{THz}$ science and its future recommended a national $\mathrm{THz}$ network to build the user community before construction for a highly stable source based on coherent synchrotron radiationy. The leading candidate is CIRCE, which would use the existing ALS injection system and reside on the roof of the booster synchrotron. Providing ultrashort pulses, it will be well suited to a wide variety of pump-probe experiments.

Storage rings, such as the ALS, are close to theoretical limits in performance. Superconducting, linac-based, high-repetition-rate FELs and ERLs promise orders of magnitude improvement in average brightness, bandwidth, and pulse duration for VUV and soft x-ray science. To develop the accelerator technology necessary for a dramatic increase in our science-reach a decade from now, the ALS is collaborating with the Berkeley Lab Accelerator and Fusion Research and Engineering divisions on ultrabright electron sources, electron-beam manipulation and conditioning by optical and other methods, techniques for producing coherent radiation, and synchronization.

\section{ACKNOWLEDGMENTS}

The authors wish to acknowledge the many members of the ALS staff and user community who contributed to the formulation of the strategic plan. This work was supported by the Director, Office of Science, Office of Basic Energy Sciences, of the U.S. Department of Energy under Contract No DE-AC02-05CH11231.

\section{REFERENCES}

1. Advanced Light Source Strategic Plan, March 2006, available on line at www-als.lbl.gov/als/ourorg/strategicplan.pdf. 